\title{
Biological classification system (BCS); with a new perspective
}

\begin{abstract}
Biopharmaceutical classification system (BCS) is a drug development tool that is based on correlation of solubility with their bioavailability in human body and allows estimation of the contributions of three major factors, dissolution, solubility, and intestinal permeability, which affect oral drug absorption from immediate release (IR) solid oral products. This mini review gives an overview of BCS with special emphasis on concept, classification, extension and Biopharmaceutics drug disposition classification system (BDDCS) with brief idea on its applications.
\end{abstract}

Keywords: biopharmaceutical classification system, solubility, bioavailability, intestinal permeability, bioequivalence, product development, BDDCS
Volume 3 Issue 4 - 2017

Vivek P Chavda, Moinuddin Soniwala

Department of Pharmaceutics, BK Mody Government Pharmacy College, India

Correspondence: Vivek P Chavda, Department of Pharmaceutics, BK Mody Government Pharmacy College, Near Ajidem, Rajkot-Bhavnagar highway, Gujarat Technological University, Rajkot-360003, Gujarat, India, Fax 028I 2384279 Email vivek7chavda@gmail.com

Received: June 03, 2017 | Published: July 25, 2017
Abbreviations: BDDCS, biopharmaceutics drug disposition classification system; IR, immediate release; BCS, biopharmaceutical classification system; BA, bioavailability; BE, bioequivalence; QBCS, quantitative biopharmaceutical classification system

\section{Introduction}

The Biopharmaceutical Classification System (BCS) has increases the applicability and validity of drug solubility and permeability in terms of research. ${ }^{1,2}$ It is now well clear that drug absorption dependent on bioavailability and bioequivalence during the drug development process provided by U. S. Food \& Drug Administration. ${ }^{3}$ The Biopharmaceutical classification system (BCS) is cogent tool for decision making during the phases of early drug development. ${ }^{4}$ The BCS was first devised in 1995 and became a standard in the regulation of bioequivalence of oral dosage forms. ${ }^{5,6}$ The BCS is a scientific framework that classifies a drug substance based on two parameters

a. Aqueous solubility

b. Intestinal permeability. ${ }^{6}$

If the absorption of the drug substance is permeation rate limited, the solubility of the drug substance will not be a governing parameter and so the in vitro dissolution study can be used to demonstrate the bioavailability $(\mathrm{BA})$ or bioequivalence $(\mathrm{BE})$ of the drug product through in vitro - in vivo correlation (IV-IVC). ${ }^{1,7}$ In BCS, each class has particularly designated rate-limiting step with possibilities for its modification so that the formulator can select and optimize a specific dosage form for the drug substance belonging to a particular class of BCS ${ }^{8,9}$ Here we are mainly focusing on some of the new concepts of BCS.

\section{Extension to BCS}

\section{Six class biopharmaceutical classification system}

Bergstrom and co-workers have dividing the drugs into six classes considering solubility, permeability and calculated surface area which is the modified version of the BCS. The solubility was allotted as "high" or "low" and the permeability was classified as "low", "intermediate," or "high". The non-polar portion of surface area of the molecule terminated in good permeability predictions. It is well suited for early development especially for optimization of the pharmacokinetic parameters. ${ }^{10}$

\section{Quantitative biopharmaceutical classification system (QBCS)}

Apart from solubility of drug in the dissolution fluid dissolution can be dependent on the amount of drug present at the site of absorption (dose) considering this fact the quantitative BCS (QBCS) was designed by Rinaki and $\mathrm{Co}-$ workers. The classification was based on the solubility: dose ratio as main parameter. According to FDA guidance for the industry, August 2000, the highest dose strength of an immediate release product should be considered for study. ${ }^{11}$ The QBCS relies on a permeability and dose/solubility ratio. Permeability estimates, ( $\mathrm{P}$ app that is the apparent permeability) were derived from Caco-2 cell studies and a constant intestinal volume content of $250 \mathrm{ml}$ was used to express the dose solubility ratio as a dimensionless quantity (q) (Table 1). Papp is the apparent permeability coefficient, which is the ratio of flux to concentration of drug in the donor compartment. Hence from it in-vitro tissue model can be used to predict in-vivo data. Apart from this the chiral conversion of the drug in the body is also considered by Gohel $\mathrm{MC}$ and $\mathrm{Co}-$ workers and gives Chirality based classification as given in Table 2 .

\section{Biopharmaceutics drug disposition classification sys- tem (BDDCS) ${ }^{12,13}$}

Chi Yuan $\mathrm{Wu}^{13}$ and co-workers in the 2005 proposed BDDCS considering factors like efflux transporters, food, absorptive transporters and renal or biliary routes of elimination on drug absorption and bioavailability. In the BDDCS, permeability is replaced by extent of metabolism and drugs are classified on the base of solubility and extent of metabolism. According to classification, if the drug is mainly eliminated by metabolism, then the drugs show high permeability. If the drug is mainly eliminated as unchanged drug by biliary or renal route, then the drugs show low permeability. Formerly, "Extensive metabolism" was defined as $\geq 50 \%$ metabolism of an oral dose in humans in vivo but now "extensive metabolism" 
mean $\geq 70 \%$ metabolism of an oral dose in vivo in humans whereas the "poor metabolism" mean excretion of $\geq 50 \%$ of the dose unchanged (Figure 1). ${ }^{14,15}$

Table I Quantitative biopharmaceutical classification system

\begin{tabular}{lll}
\hline BCS class & Papp* $(\mathbf{c m} / \mathbf{s e c})$ & q* \\
\hline I & Papp $>10-5 \mathrm{~cm} / \mathrm{sec}$ & $\mathrm{q} \leq 0.5$ \\
II & Papp $>10-5 \mathrm{~cm} / \mathrm{sec}$ & $\mathrm{q}>$ I \\
III & Papp $<2$ C $10-6 \mathrm{~cm} / \mathrm{sec}$ & $\mathrm{q} \leq 0.5$ \\
IV & Papp $<2$ C $10-6 \mathrm{~cm} / \mathrm{sec}$ & $\mathrm{q}>$ I
\end{tabular}

*Papp- apparent permeability and q- Dose/solubility.

Table 2 Chirality and BCS classification

\begin{tabular}{llll}
\hline Class & Solubility & Permeability & Chiral conversion* \\
\hline I & High & High & A Hight \\
& & B Low€ \\
II & Low & High & A High \\
& & & B Low \\
III & High & Low & A High \\
& & & B Low \\
IV & Low & Low & A High \\
& & & B Low \\
\hline
\end{tabular}

*Chiral Conversion- an active form of drug is converted into an inactive form in blood stream. For racemic drugs, the fraction of dose of active enantiomer reaching the receptor site is more relevant for pharmacological response.

$£$ High- higher amount of active enantiomer is converted to inactive form.

$€$ Low- lower amount of active enantiomer is converted to inactive form.

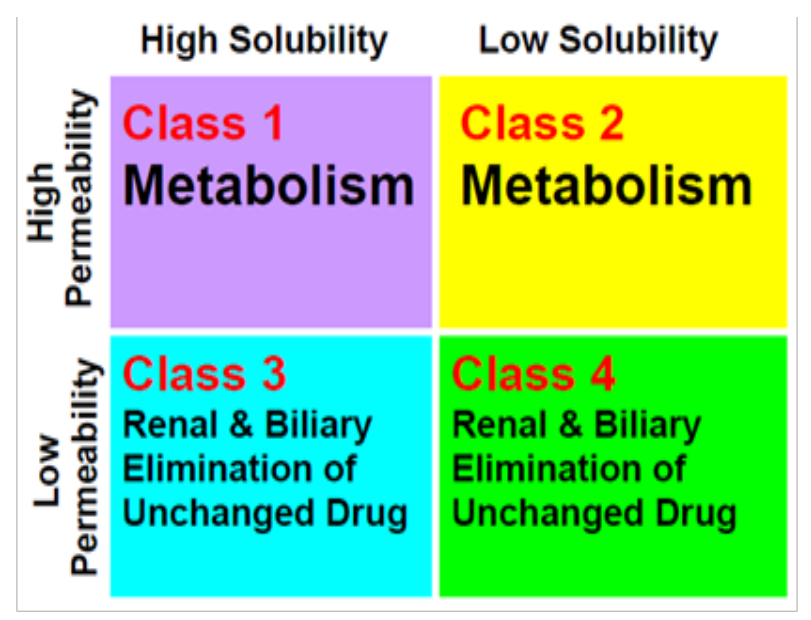

Figure I Major route of drug elimination.

\section{BCS - the future prospect}

Since the birth of the BCS there are lot many revision of the BCS guidelines which will result in increased chances of applicability in development of drug with desired characteristics. A special emphasis should be given to the use of BCS in determination of the rate limiting step in the process of oral absorption during early phases of drug development and provision of important information to formulation scientists about the overall drug development process. BCS is not only a regulatory tool but also useful for the early development of molecule. ${ }^{16}$ Substantial differences of bio-waiver dossiers and respective assessments contribute to the impression that a common understanding is lacking on a successful use of the BCS concept to support.

\section{Acknowledgements}

None.

\section{Conflict of interest}

The author declares no conflict of interest.

\section{References}

1. Amidon GL, Lennernäs H, Shah VP, et al. A theoretical basis for a biopharmaceutic drug classification: The correlation of in vitro drug product dissolution and in vivo bio-availability. Pharm Res. 1995;12(3):413420 .

2. Series CS. Biopharmaceutics Drug Classification and International Drug Regulation. Tokyo: Seminars and Open Forums; 1997. p. 1-187.

3. Waterbeemd HV, Testa B. Drug Bioavailability: Estimation of Solubility, Permeability, Absorption and Bioavailability. Weinheim: Wiley-VCH; 2009.

4. $\mathrm{Ku}$ MS. Use of the Biopharmaceutical Classification System in early drug development. AAPS J. 2008;10(1):208-212.

5. Meyer MC, Straughn AB, Jarvi EJ, et al. The bioinequivalence of carbamazepine tablets with a history of clinical failures. Pharm Res. 1992;9(12):1612-1616.

6. CDER/FDA Waiver of in vivo bioavailability and bioequivalence studies for immediate release solid oral dosage forms based on a biopharmaceutics classification system. USA: Guidance for industry; 2000. p. 1-17.

7. Biopharmaceutics Classification System Guidance Office of Pharmaceutical Science CF. 2006

8. Ahuja A KR, Ali J. Dosage Form Design. 1st ed. Delhi: Birla Publications Pvt Ltd; 2004. p. 3-11.

9. Yu LX CA, Hussain AS. Feasibility study of intrinsic dissolution rate as an alternative method to determine BCS solubility membership. AAPS Annual Meeting, USA; 2000.

10. Dash V, Kesari A. Role of Biopharmaceutical Classification System in Drug Development Program. Journal of Current Pharmaceutical Research . 2011;5:28-31.

11. Dokoumetzidis A, Valsami G, Macheras P. Modelling and Simulation in Drug Absorption Processes. Xenobiotica. 2007;37(10-11):1052-1065.

12. Tubic-Grozdanis M, Bolger MB, Langguth P. Application of gastrointestinal simulation for extensions for biowaivers of highly permeable compounds. AAPS J. 2008;10(1):213-226.

13. Wu CY, Benet LZ. Predicting drug disposition via application of BCS: transport/absorption/elimination interplay and development of a biopharmaceutics drug disposition classification system. Pharm Res. 2005;22(1):11-23.

14. Chen ML, Amidon GL, Benet LZ, et al. The BCS, BDDCS, and reglatory guidances. Pharm Res. 2011;28(7):1774-1778.

15. BDDCS: Biopharmaceutical Drug Disposition Classification System. Solvo Biotechnology.

16. Lennernäs $H$, Abrahamsson B. The use of biopharmaceutics classification of drugs in drug discovery and development: current status and future extensions. J Pharm Pharmacol. 2005;57(3):273-285. 